\title{
Comparison of simulated and observed beam profile broadening in the Proton Storage Ring and the role of space charge
}

\author{
J. D. Galambos, S. Danilov, D. Jeon, J. A. Holmes, and D. K. Olsen \\ Oak Ridge National Laboratory, Oak Ridge, Tennessee 37831 \\ F. Neri and M. Plum \\ Los Alamos National Laboratory, Los Alamos, New Mexico 87545
}

(Received 18 March 1999; published 13 March 2000)

\begin{abstract}
Transverse beam profiles are observed to broaden with increasing intensity in the Proton Storage Ring at the Los Alamos Neutron Scattering Center. Measured profiles are simulated with an $\mathrm{H}^{-}$injection model that includes a 2D particle-in-cell space charge calculation. Inclusion of space charge effects in the simulation improves the agreement between the experimentally observed profiles and the calculated profiles. The comparisons are made for a range of injected intensities.

PACS numbers: 41.85.-p, 29.27.Bd
\end{abstract}

\section{INTRODUCTION}

Space charge effects will be an important factor in determining beam profiles and halo generation in highintensity, low-energy storage rings and synchrotrons such as the Spallation Neutron Source (SNS) [1]. Of particular concern is space charge induced halo generation, which can activate the ring to levels above that allowed for hands-on maintenance. In order to have confidence in predicting the magnitude of space charge effects, it is important to benchmark calculations to experimental results. Consequently, we present comparisons of simulated and experimentally measured transverse beam profiles at the end of injection cycles, for increasing levels of beam intensity in the Los Alamos Neutron Science Center (LANSCE) Proton Storage Ring (PSR). The simulations were conducted with a new code we are developing that includes realistic injection scenarios with closed orbit bumps, foil effects, rf voltage, etc., as well as a particlein-cell (PIC) transverse space charge model. Experiments were performed at the PSR [2] in which the beam transverse profiles were measured with a wire scanner in the extraction beam line. Inclusion of space charge effects in the simulation greatly improves the agreement between the shape of the calculated profiles and the experimentally measured profiles. Additional details of this comparison are given in Ref. [3]. This comparison differs from a previous study of transport simulations and PSR measurements [4] in which measured activation levels around the ring were compared to calculated profiles without the inclusion of the transverse space charge force.

\section{EXPERIMENTS}

Experimental beam profiles were measured at the PSR, which is a storage ring, with $\mathrm{H}^{-}$injection from an $800 \mathrm{MeV}$ linac. Injection into the ring is a multiturn process through a stripping foil. Most of the injected linac $\mathrm{H}^{-}$beam is stripped to $\mathrm{H}^{+}$at the foil, and subsequently oscillates about the ring closed orbit. The foil effectively acts as a source of ring particles, and as such the ring injection process violates the Liouville condition. The linac emittance (see Table I) is much smaller than the final ring emittance, and, by moving the closed orbit during the injection process, it is possible to "paint" a ring distribution. When the linac spot on the foil is offset from the ring closed orbit, the injected particles produce an annular ring, or "smoke ring" distribution in phase space after several turns. The projection of this distribution onto the vertical plane is a hollow profile. Figure 1 shows the transverse phase space and real space distributions at the end of injection bump (calculated without space charge) for the parameters in Table I corresponding to the case with no closed orbit bump. The vertical phase space distribution is very narrow and hollow due to the large offset between the foil and the closed orbit, and the small linac beam spot size relative to this offset. Since the ring $\beta_{x}<\beta_{y}$ at the foil, the horizontal offset is smaller, and, because the injected beam size tends to be large compared to the horizontal closed orbit offset, the horizontal distribution is less hollow. The horizontal distribution is also broadened since the foil is located in a region of the ring with dispersion, so the linac beam momentum spread produces a spread in the injected horizontal ring distribution. The extraction process is Liouvillian and does maintain the accumulated ring emittance.

Injection was carried out for $825 \mu \mathrm{s}$, or 2305 turns, for three different intensity levels, both with and without a vertical closed orbit bump. Subsequently, the beam was extracted in a single turn and transported to a wire scanner beam profile diagnostic in the extraction beam line. The purpose of this study was to observe the effect of the transverse space charge force on the beam profile shape. To this end, a series of measurements was completed in succession in which the injected particle intensity was varied by changing the interval of injection 
TABLE I. Input parameters used to model the PSR injection scenario.

Injection period

Injection turns

Injected particles/turn

Linac beam location relative to closed orbit

Horizontal closed orbit bump at foil

Vertical closed orbit linear bump at foil

rf

Ring injection $\beta_{x}, \alpha_{x}, D_{x}$

Linac $\beta_{x}, \alpha_{x}$

$$
\beta_{y}, \alpha_{y}
$$

$$
\beta_{y}, \alpha_{y}
$$

Linac horizontal distribution bi-Gaussian

Linac vertical distribution bi-Gaussian

Linac longitudinal distribution

$825 \mu \mathrm{s}$

2305

$1.3 \times 10^{10}$

Horizontal: $3.85 \mathrm{~mm},-0.95 \mathrm{mrad}$; Vertical: $16.64 \mathrm{~mm}, 2.68 \mathrm{mrad}$ None

None or $12 \rightarrow 0 \mathrm{~mm}$ and $1.65 \rightarrow 0 \mathrm{mrad}$

Single harmonic, linear ramp from 8 to $17 \mathrm{kV}$ during injection

$$
2.77 \mathrm{~m}, 0.63,1.42 \mathrm{~m}
$$

$10.93 \mathrm{~m},-1.43$

$1.25 \mathrm{~m}, 0$

$3.20,0$

$30 \%$ with $\varepsilon_{2 \sigma}=0.33 \pi \mathrm{mm} \mathrm{mrad}$

$70 \%$ with $\varepsilon_{2 \sigma}=1.0 \pi \mathrm{mm} \mathrm{mrad}$

$40 \%$ with $\varepsilon_{2 \sigma}=0.33 \pi \mathrm{mm}$ mrad

$60 \%$ with $\varepsilon_{2 \sigma}=1.0 \pi \mathrm{mm} \mathrm{mrad}$

50 bi-uniform bunches separated by $4.97^{\circ}$ (or $4.94 \mathrm{~ns}$ ) each. Each bunch has $70 \%$ within $0.025^{\circ}$ and $8 \mathrm{MeV}$, $30 \%$ within $1.0^{\circ}$ and $32 \mathrm{MeV}$
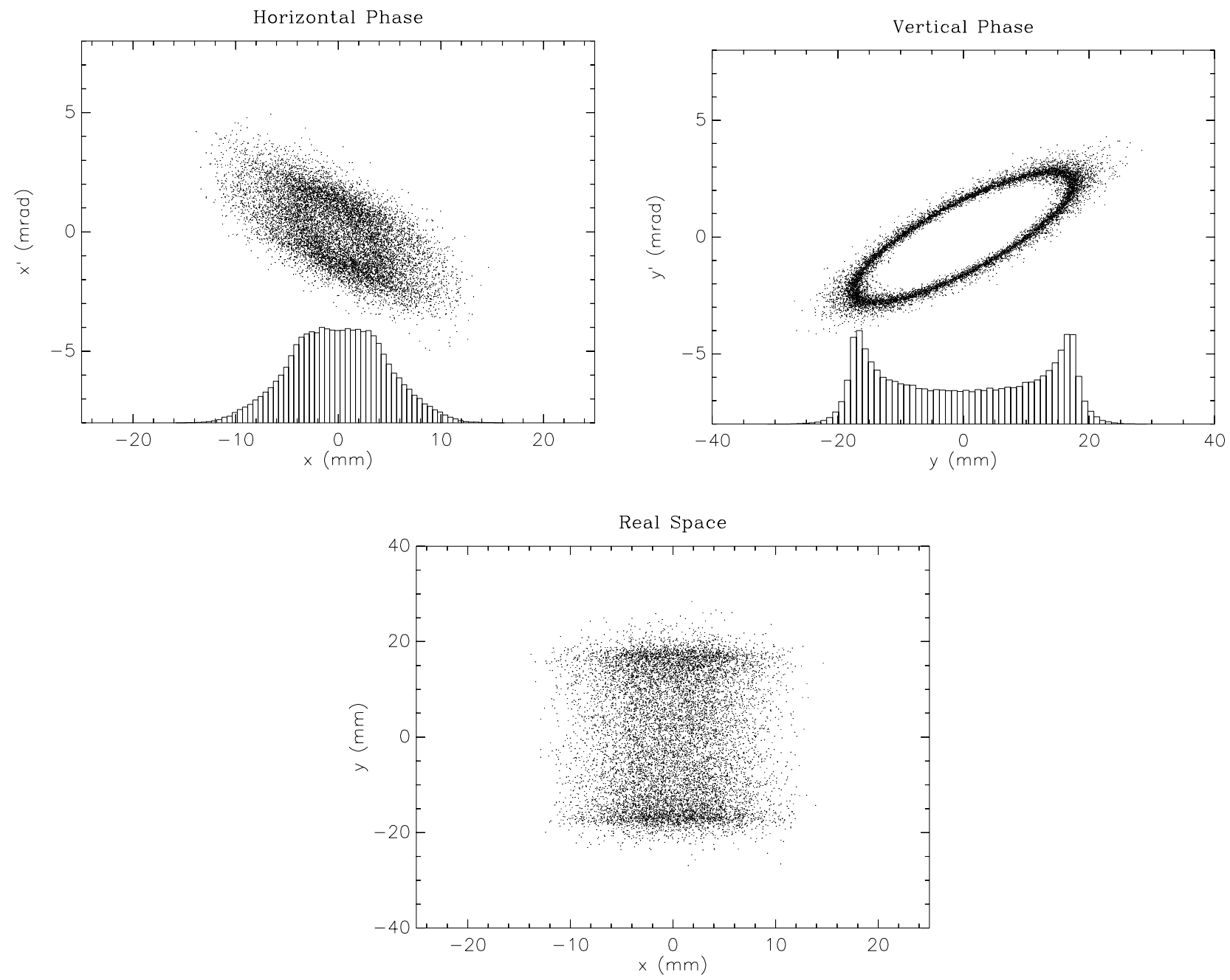

FIG. 1. Calculated phase space distributions at the end of injection, for the case with no vertical bump during injection and no space charge. 
turns. Measurements were completed with linac beam injected into the storage ring every turn (countdown $=$ 1 ), every other turn (countdown $=2$ ), and every fourth turn (countdown =4). All other linac, injection, and PSR parameters, such as ion source strength, closed orbit bumps, etc., were held fixed. The measurements were performed on October 28, 1998, and the beam profile results shown here were obtained from the ROWS02 wire scanner diagnostic.

Comparing the areas under the beam profiles for various countdown levels provides a check on wire scanner data. For a perfectly linear response, the area under a countdown $=2$ case would be one-half that for the corresponding countdown $=1$ case. For the wire scanner data shown here, relative to the countdown $=1$ cases the response is below a linear response by $2 \%-3.7 \%$ for the countdown $=2$ cases and by $8 \%-11 \%$ for the countdown $=4$ cases. This level of accuracy is consistent with previous experience with the wire scanner system. There is no systematic difference between the horizontal and vertical responses. Additionally, for equivalent countdowns the area under the observed profiles should be the same for the cases with and without a vertical bump during injection. However, there are variations from $0.3 \%$ to $3.3 \%$ in the areas for equivalent countdowns. In the comparisons shown below, the calculated profiles are normalized to have the same area as for the corresponding experimentally observed profiles.

\section{SIMULATIONS}

The ORBIT particle-tracking code, with injection and space charge, used to perform the calculations is undergoing development for the SNS project and is described in Ref. [5]. The injection parameters are described in detail in Table I. Both transverse and longitudinal space charge forces are included in the simulations, but transverse space charge effects are the primary subject of this paper. In particular, Hill's equation for the transverse motion for a single particle is given by

$$
\left\{\begin{array}{l}
x \\
y
\end{array}\right\}^{\prime \prime}=F_{x, y}^{\mathrm{lin}}+F_{x, y}^{\mathrm{sc}}+F_{x, y}^{\mathrm{n} 1},
$$

where the space charge force on the particle from the distribution of particles is given by

$$
F_{x, y}^{\mathrm{sc}}=\left\{\frac{2 Z^{2} r_{p} \lambda}{\gamma^{3} \beta^{2} A N}\right\} \sum_{i} \frac{\left\{\begin{array}{l}
x-x_{i} \\
y-y_{i}
\end{array}\right\}}{\left(x-x_{i}\right)^{2}+\left(y-y_{i}\right)^{2}} .
$$

The term $F_{x}^{\text {lin }}$ contains the single particle linear transport, including dispersion, through drifts, bends, normal and skew quadrupoles, and solenoids. The term $F_{x}^{\mathrm{n} 1}$ contains the single-particle nonlinear magnet forces, including chromaticity, fringe fields, field errors, and higher order multipoles. Although the nonlinear magnet forces have recently been implemented, they were not used in the present calculations.

Equations (1) and (2) are solved using a 2D PIC method employing a bilinear distribution of macroparticles to the nodes of a rectangular grid and then using a fast-Fouriertransform (FFT) method on that grid to approximate the full nonlinear space charge force. In particular, the space charge forces are obtained from a convolution of the transformed charge density and unit force function on the grid, as described in Ref. [6]. The beam is tracked for the full injection cycle, with the parameters described in Table I. After 2305 injection turns, or $825 \mu \mathrm{s}$, the beam is mapped to the ROWS02 wire scanner using a linear transfer matrix and then binned transversely with a resolution chosen to match the wire scanner step size.

For the calculations shown herein, 50 macroparticles per turn were injected, resulting in 115250 macroparticles at the end of injection. A $128 \times 128$ grid was used in the transverse plane for the PIC model, and 197 space charge kicks were applied per turn $(\sim 45 \mathrm{~cm}$ longitudinal distance per kick). This corresponds to about 60 kicks per horizontal betatron oscillation, about 90 kicks per vertical betatron oscillation, and 20 kicks per FODO cell. Second order symplectic integration is employed. Sensitivities to the numerical parameters are described in Ref. [3]. The resultant profile shapes are insensitive to grid size and macroparticle number for grid sizes $\geq 64 \times 64$ and macroparticle numbers $>5 \times 10^{4}$. No additional smoothing is used beyond that inherent in the binning on the PIC grid and use of an FFT approximation for the force. Sixtyfour bit precision is used, but sensitivities indicate that use of 32 bit precision does not have an impact on the bulk effects of the beam. The space charge force is the only nonlinear contribution to the beam transport in the simulation. In these calculations neither magnet errors nor chromatic nor transverse impedance effects were included. The calculations required about $10 \mathrm{~h}$ on a $533 \mathrm{MHz}$ alpha workstation.

\section{COMPARISON OF SIMULATION AND EXPERIMENT}

Because the absolute transverse location of the wire scanner was not precisely known, the centers of the calculated distributions are adjusted to match that of the experimental data. Also, as previously discussed, the vertical scale of the calculated distributions is adjusted so that the area under the calculated profiles matches that from the wire scanner data. The width and shape of the calculated distribution are not adjusted. Comparisons are made for two injection scenarios, both with and without a vertical closed orbit bump at the foil. The PSR does not have bump magnets to paint in the horizontal direction, but a horizontal spread is provided through the injected beam energy distribution since there is a dispersion of $1.4 \mathrm{~m}$ at the foil location. 


\section{A. Injection with a closed orbit bump}

Figure 2 shows the comparison between both horizontal and vertical measured and calculated beam profiles for three beam intensities, for the case using a dynamically painted vertical closed orbit bump at the foil during injection. The calculated horizontal profiles are in good agreement with the measured profiles, and the effect of increasing beam intensity on the shape of the distribution is small. The vertical beam profiles are observed to broaden and to fill in, becoming less hollow, with increasing intensity, for both the calculated and experimental distributions. The agreement is closer at low intensities than at the high intensity level of $3 \times 10^{13}$ protons. At the full intensity there is still a slight hollowness in the calculated distribution, whereas the experimental profile is centrally peaked. In addition, the measured distributions are slightly broader than the calculated distributions.

The inclusion of transverse space charge forces in these calculations contributes significantly to the goodness of the profile comparisons shown in Fig. 2. For example, Fig. 3 shows the comparison of the experimental and calculated profiles at the highest intensity. In this figure, calculated profiles are shown both with and without transverse space charge. There is little difference in the horizontal profile shape calculated with and without space charge, consistent with the small impact on the experimentally observed horizontal profiles in Fig. 2. On the other hand, the vertical beam profile calculated without space charge forces is significantly more peaked than either the calculation with space charge or that observed experimentally. A measure of the error between the calculated and experimentally observed profiles is the rms error normalized to the average experimental profile, namely,

$$
R_{\text {error }}=\frac{\sqrt{\sum_{i}\left(f_{i}^{\exp }-f_{i}^{\text {calc }}\right)^{2} N}}{\sum_{i} f_{i}^{\exp }},
$$

where $f_{i}^{\text {exp }}$ is the wire scanner profile at point $i, f_{i}^{\text {calc }}$ is the corresponding calculated profile magnitude, and the sum is over all $N$ wire scanner points. The error using this measure is $12 \%$ with space charge and $37 \%$ without space charge included in the calculations. While not entirely accounting for the experimentally observed beam broadening and filling in of the central part of the profile, the transverse space charge model does reproduce much of the observed beam broadening.
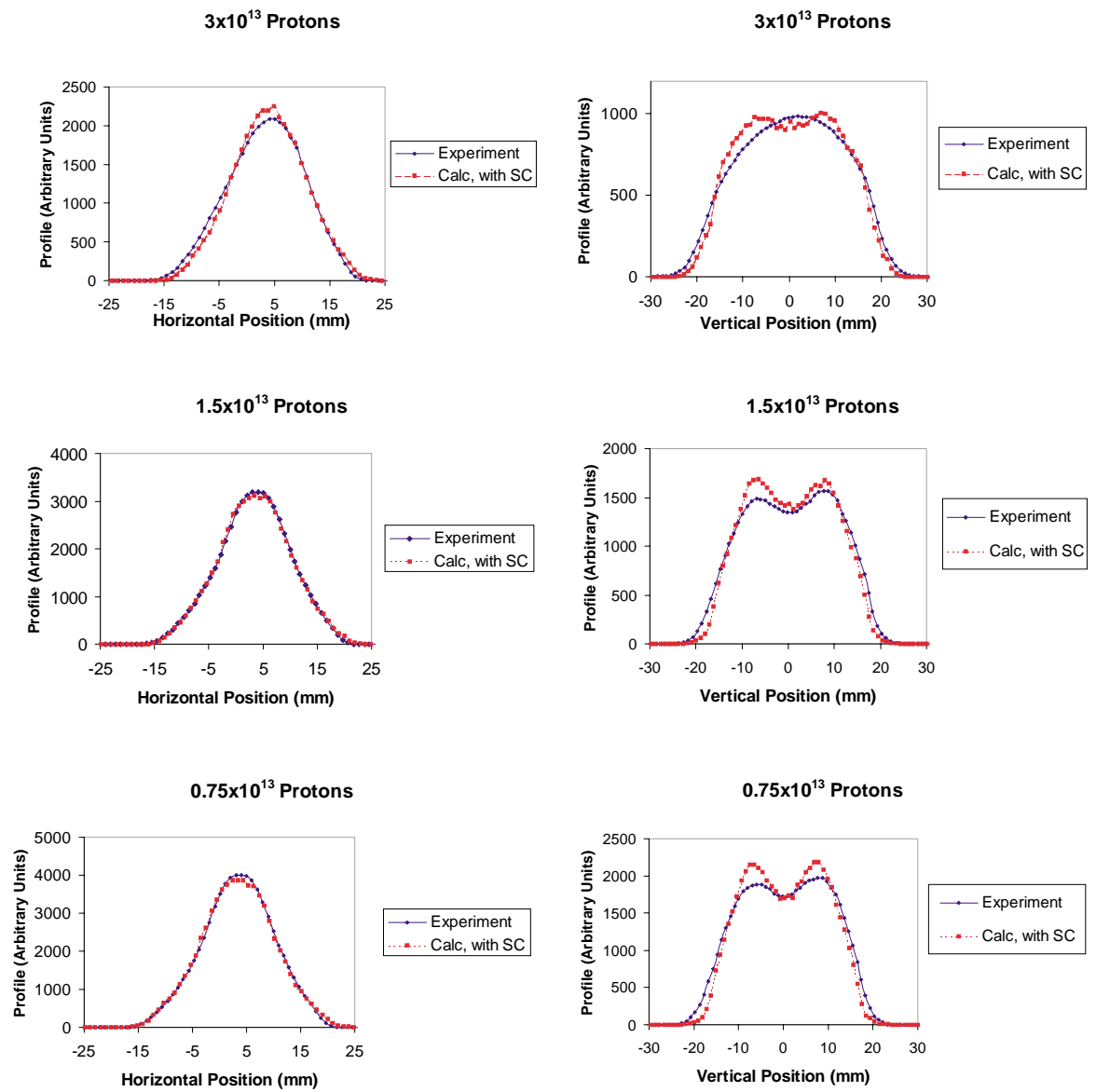

FIG. 2. (Color) Comparison of the calculated and measured horizontal and vertical distributions for three accumulated beam intensities, with vertical painting. Space charge forces are included in the calculations. 

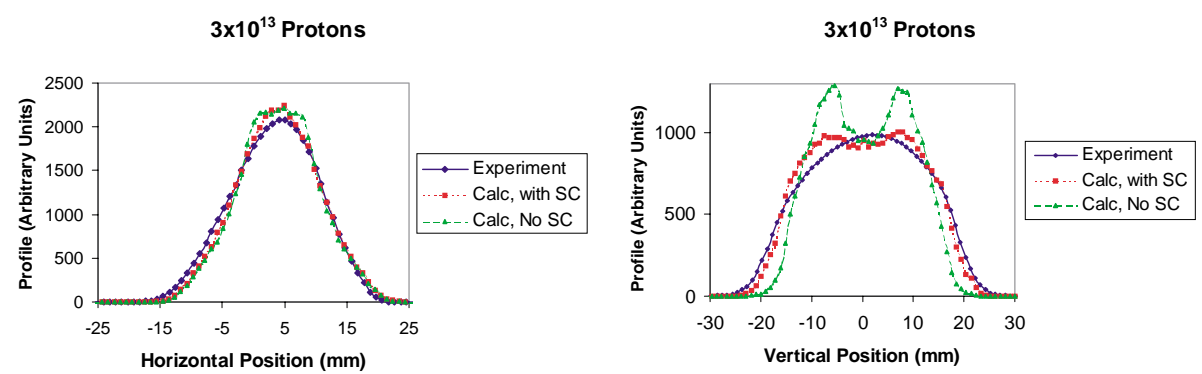

FIG. 3. (Color) The effect of the space charge force on the calculated beam profiles, for the case using a painted vertical bump during injection and at the highest intensity.

\section{B. Injection without a closed orbit bump}

In this case, neither horizontal nor vertical dynamic orbit bumps are used during the injection. The linac beam offset from the closed orbit is fixed throughout the entire injection scenario resulting in the injection of a hollow "smoke ring" vertical distribution in phase space, as described in Sec. II. Figure 4 shows the profile comparisons for the three injected intensities. As before, the agreement for the horizontal profile is good and there is not a dramatic dependence of the profile shape on intensity. The calculated horizontal profile slightly underestimates the broadening at high intensities. The beam vertical profile is more strongly affected by intensity. The center of the vertical profile fills in noticeably with increasing intensity for both the measured and calculated distributions. However, the calculated profiles are again slightly less broad than the measured distributions. Figure 5 shows the results at full intensity, calculated both with and without the space charge force.
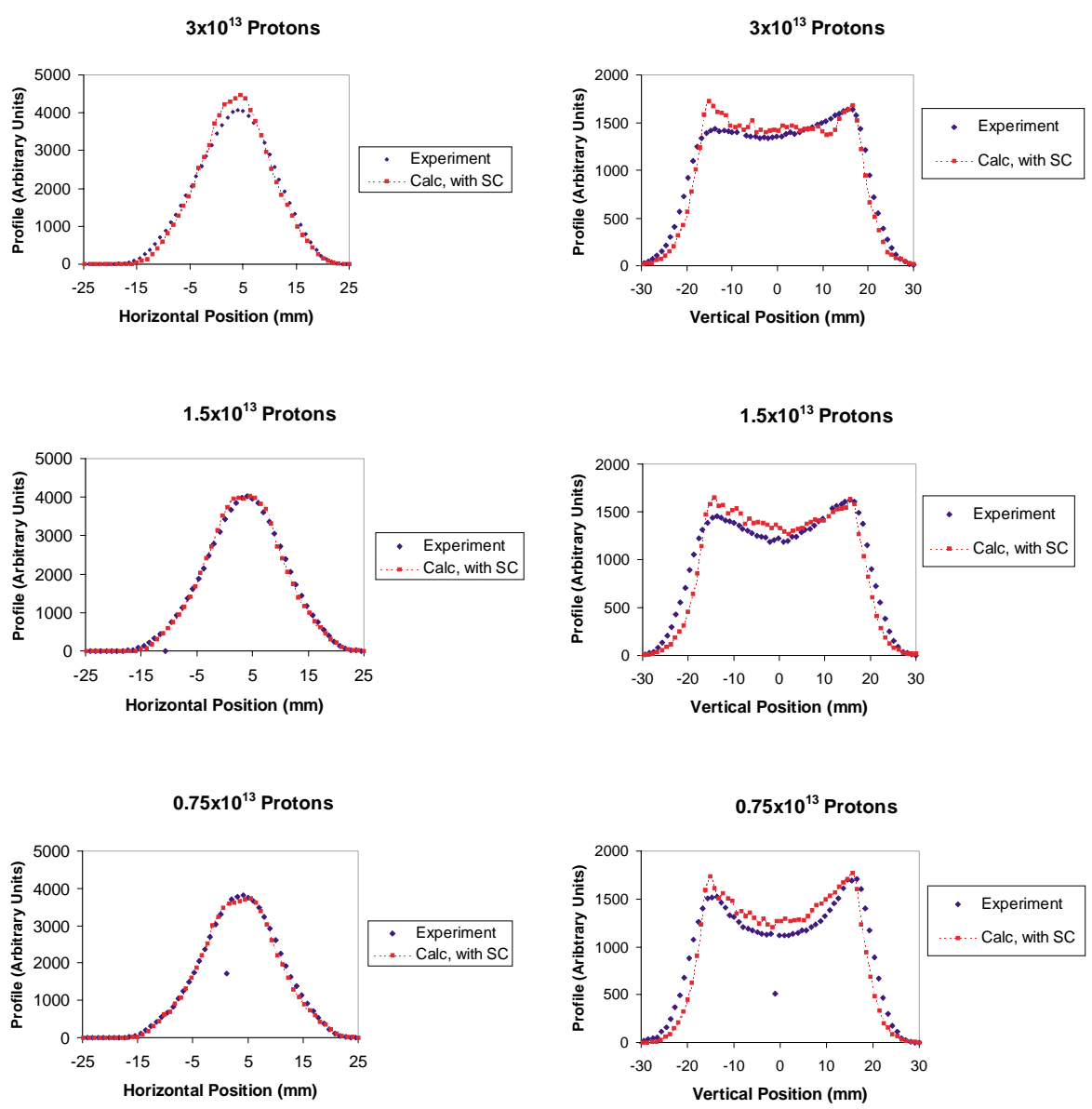

FIG. 4. (Color) Comparison of the calculated and measured horizontal and vertical distributions for three accumulated beam intensities, with no vertical painting. Space charge forces are included in the calculations. 

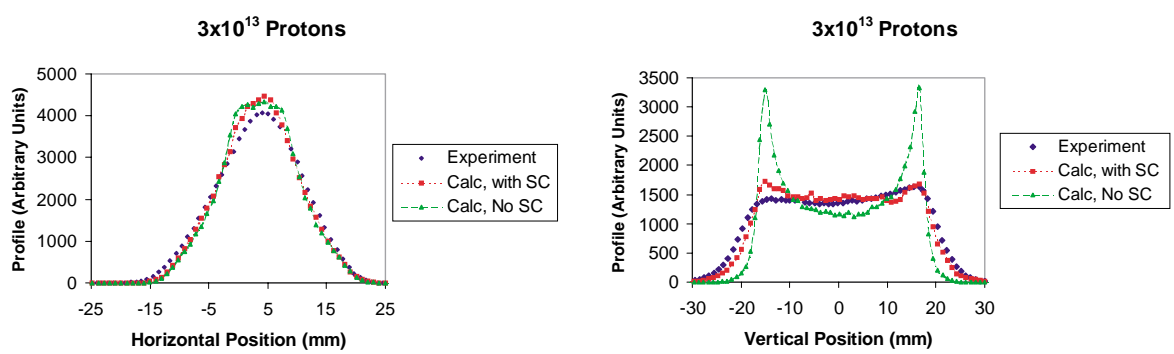

FIG. 5. (Color) The effect of the space charge on the calculated beam profiles, for the case with no closed orbit bumps during injection and at the highest intensity.

Without the space charge force the calculation predicts a narrow, hollow distribution. This distribution is peaked at the point corresponding to the linac beam offset, and the peak width corresponds to the linac beam size. Neither the experiment nor the calculation with space charge resemble this profile. There is a significant filling in of the hollow profile and broadening of the distribution. The transverse space charge model predicts most of the observed beam broadening. The error between the calculated and observed profiles using the measure defined above $\left(R_{\text {error }}\right)$ is $15 \%$ with space charge included and $57 \%$ without space charge included.

Both with and without the vertical bump included during the injection, the observed horizontal profiles maintain their overall shape and the vertical profile distribution broadens with increasing intensity. Figure 6 shows the characteristic beam horizontal and vertical sizes versus intensity at the end of injection for the distributions shown in Figs. 2 and 4. The beam size is taken here as the width containing $99 \%$ of the area under the profile. This figure shows the lack of intensity dependence of the horizontal beam size for both the experimentally observed and calculated profiles. For the case without a vertical bump, the experimental vertical beam size increases by about $6 \%$ and the calculated beam size with space charge increases by about $8 \%$ as the beam intensity increases from $0.75 \times 10^{13}$ to $3 \times 10^{13}$. With the vertical bump, the increase in the experimentally observed beam size over this intensity variation is $16 \%$ and the calculated beam with space charge size increases $13 \%$. In both cases there is a systematic 3\%-9\% underestimate of the vertical beam size for the calculation with space charge. Without inclusion of space charge in the calculation there is no intensity effect on the beam size, and the underestimate of the calculated beam size compared to the experimental beam size varies from $7 \%$ at low intensity to $23 \%$ at high intensity. If the vertical beam broadening with intensity were due to nonlinearity of the wire-scanner response, the horizontal profiles should also broaden with increasing intensity, since the response of the horizontal scanner is similar to that of the vertical scanner. However, there is no observed broadening in the experimental horizontal profile.
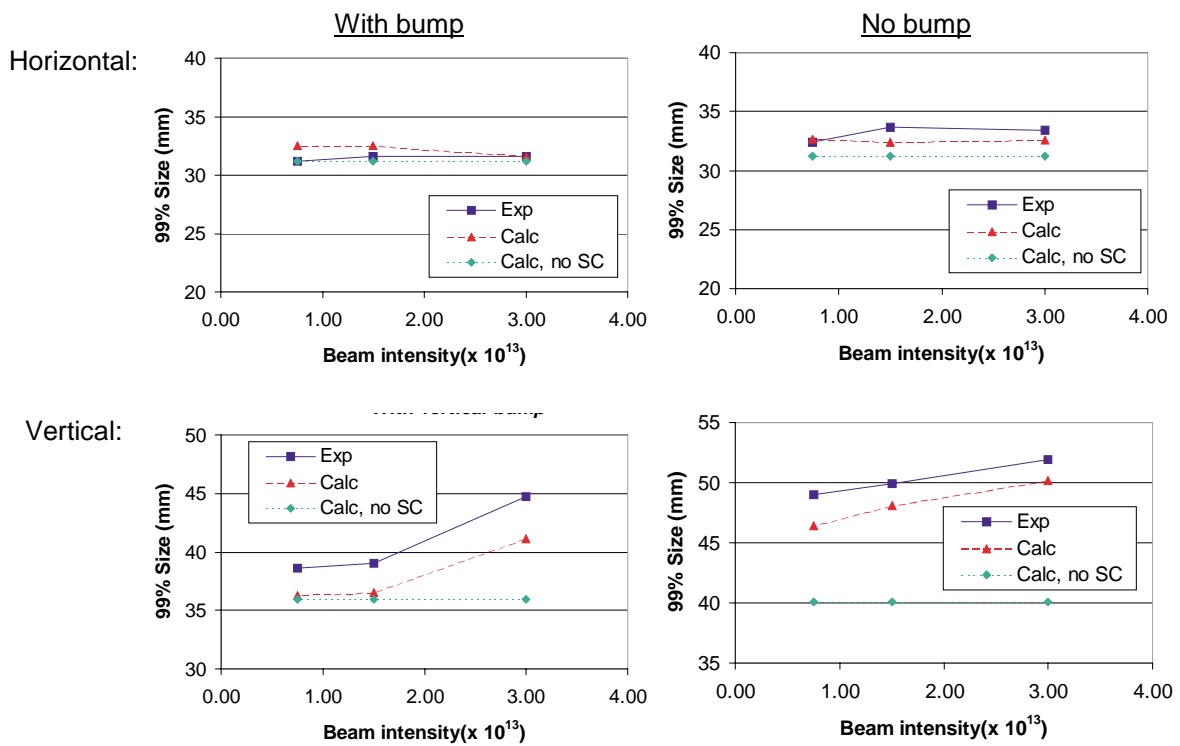

FIG. 6. (Color) Horizontal and vertical $99 \%$ beam size versus beam intensity for cases with and without the vertical injection bump. 


\section{DISCUSSION}

Observed beam profiles from high-intensity beams at the PSR are compared to those from simulations. Agreement between the measured and calculated transverse profiles is significantly improved when space charge effects are included. There is a noticeable broadening of the vertical distribution with increasing beam intensity in both the experimental measurements and the calculated profiles that include space charge effects. The primary intent of this paper is to present the comparison of the numerically obtained profiles with the experimentally observed profiles. While a full explanation of the mechanisms behind the observed broadening is not the intent of this paper, a few simple explanations are made below.

The horizontal profiles are less affected by space charge than the vertical profiles for several reasons. First, the lattice focusing is stronger in the horizontal direction than in the vertical direction $\left(\nu_{x}=3.17, \nu_{y}=2.14\right)$. Second, there are stronger density gradients in the vertical direction, due to the nature of the injected beam, as discussed in Sec. II and shown in Fig. 1. Finally, there are coherent beam moment oscillations in the vertical direction which are evident in the calculations. Figure 7 shows horizontal and vertical beam moments calculated over the last three injection turns, for the case with $3 \times 10^{13}$ injected particles and with a vertical closed orbit bump. These moments have been normalized to remove the lattice effects; i.e., the quadrupole moments are

$$
\frac{\left\langle(x-\bar{x})^{2}\right\rangle}{\beta_{x}} \text { and } \frac{\left\langle(y-\bar{y})^{2}\right\rangle}{\beta_{y}},
$$

and the octupole moments are

$$
\frac{\left\langle(x-\bar{x})^{4}\right\rangle}{\beta_{x}^{2}} \text { and } \frac{\left\langle(y-\bar{y})^{4}\right\rangle}{\beta_{y}^{2}} .
$$
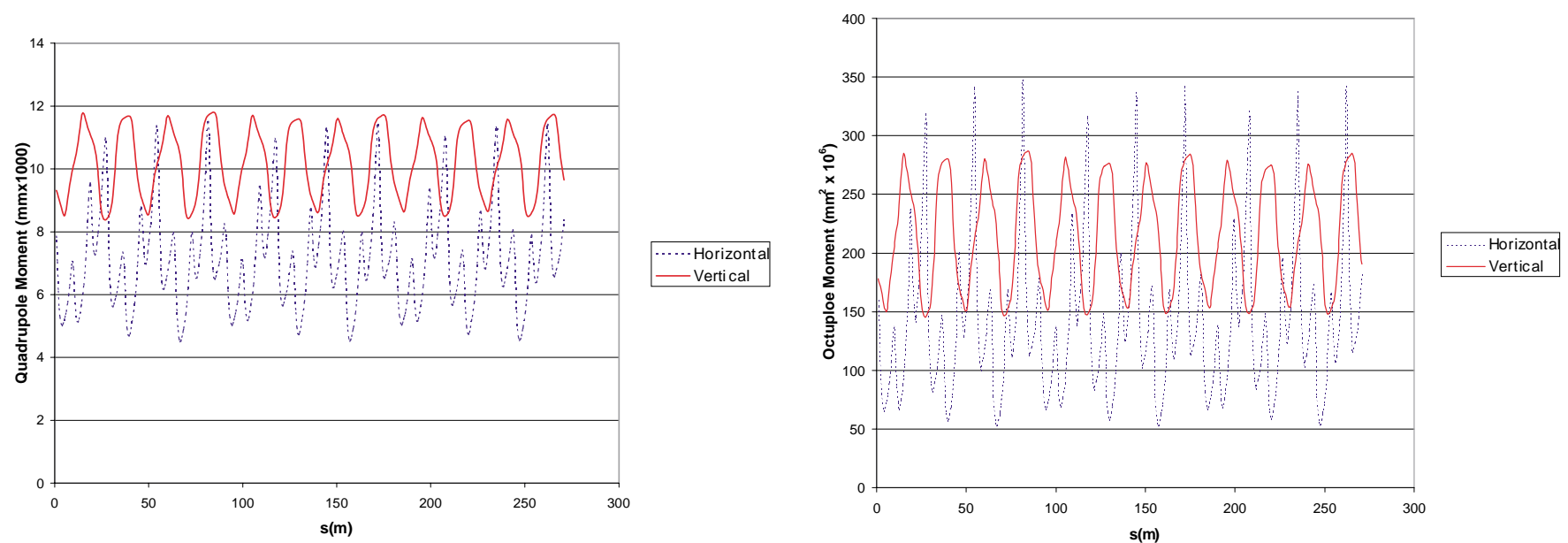

FIG. 7. (Color) Vertical and horizontal beam quadrupole and octupole moments versus longitudinal position over the last three turns, for the case with $3 \times 10^{13}$ injected particles, with a vertical bump, and with space charge. Longitudinal position $s=0 \mathrm{~m}$ corresponds to the beginning of turn 2303, and position $270.8 \mathrm{~m}$ corresponds to the end of turn 2305 .
Well-defined vertical quadrupole and octupole beam oscillations are evident, arising from the space charge forces. The space charge induced beam quadrupole oscillation can drive a parametric resonance, which can cause halo generation and beam spreading [7], and may contribute to the beam spreading seen here. The coupling resonance $\left(2 \nu_{x}-2 \nu_{y}=2\right)$ can be driven by the octupole oscillation. The horizontal moment behavior is much less regular. Also, these oscillations are not observed in the calculations performed without space charge.

While the experimentally observed beam broadening is better matched when space charge effects are included, these calculated profiles still slightly underestimate the amount of edge profile broadening at high intensities, particularly in the vertical direction. This may be due to uncertainties in the experimental measurements. For example, the linac intensity may be up to $10 \%$ larger than assumed in the simulation, and the vertical linac position relative to the closed orbit could be larger by up to $10 \%$ than the assumed value of $16.6 \mathrm{~mm}$. Also, as previously mentioned, the wire scanner response deviates from a linear response by $\sim 10 \%$ for the countdown $=4$ cases compared to the countdown $=1$ cases. The differences between observed and calculated profiles may also be caused by physics effects not included in the calculation, for example, magnet or alignment errors and wall impedance effects. Coupling of the space charge with machine errors could cause some of the experimentally observed intensity dependent beam broadening. Understanding these remaining differences is the subject of future work.

Nevertheless, the comparison of the calculated profiles with experimentally observed profiles improves significantly when space charge effects are included. The fact that the observed beam broadening increases with intensity suggests that space charge may play a role. While the present experimental measurements do not provide 
sufficient intensity resolution to benchmark the calculation of beam halo at the level required in the SNS design, one part in $10^{4}$, the comparison does increase confidence in the capability to predict the central beam distributions.

\section{ACKNOWLEDGMENTS}

Thanks are due to R. Macek of LANL for providing the opportunity to observe PSR operations, which led to this work. Also, thanks are due to A. Luccio and J. Beebe-Wang of BNL for assistance in developing the ORBIT code. We acknowledge the support of the U.S. DOE, Division of Materials Science, under Contract No. DE-AC05-96OR22464 with LMER Corp. for ORNL. This work has benefited from the use of the Los Alamos Neutron Science Center at the Los Alamos National Laboratory. This facility is supported by the U.S. Department of Energy and operated by the University of California under Contract No. W-7405-ENG-36.

[1] Oak Ridge National Laboratory Report No. NSNS/CDR1, 1997; http://www.ornl.gov/〜nsns/CDRDocuments/ CDR.html.
[2] D. H. Fitzgerald, A. Ahn, B. Blind, M. Borden, R. Macek, F. Neri, M. Plum, C. Rose, H. Thiessen, C. Wilkinson, and M. Zumbro, in Proceedings of the 1997 Particle Accelerator Conference, Vancouver, Canada (IEEE, Piscataway, NJ, 1998), p. 1012; http://www.triumf.ca/ pac97/papers/pdf/9W021.PDF.

[3] J. Galambos, S. Danilov, D. Jeon, J. Holmes, D. Olsen, F. Neri, and M. Plum, Oak Ridge National Laboratory, SNS/ORNL/AP Tech. Note No. 009, 1998.

[4] C. Wilkinson, F. Neri, D. Fitzgerald, B. Blind, R. Macek, O. Sander, and H. Thiessen, in Proceedings of the 1997 Particle Accelerator Conference, Vancouver, Canada (Ref. [2]), p. 1015; http://www.triumf.ca/pac97/ papers/pdf/9W022.PDF.

[5] J. Galambos, S. Danilov, D. Jeon, J. Holmes, D. Olsen, J. Beebe-Wang, and A. Luccio, in Proceedings of the 1999 Particle Accelerator Conference, New York (IEEE, Piscataway, NJ, 1999); http://ftp.pac99.bnl.gov/Papers/ Wpac/THP82.pdf.

[6] J. Holmes, J. Galambos, D. Jeon, D. Olsen, and J. Cobb, in Proceedings of the International Computational Accelerator Physics Conference, Monterey, CA, 1998 (unpublished).

[7] R. L. Gluckstern, Phys. Rev. Lett. 73, 1247 (1994). 